\title{
Absence of binocular interaction between spatial and color attributes of visual stimuli*
}

\author{
RAY OVERं $ं$ NIGEL LONG, and WILLIAM LOVEGROVE \\ Liniversity of Queensland. St. Lucia. Australia 4067
}

\begin{abstract}
The hypothesis that induction of the McCollough effect (spatially selective color aftereffects) entails adaptation of monocularly driven detectors tuned to both spatial and color attributes of the visual stimulus was examined in four experiments. The McCollough effect could not be generated by displaying contour information to one eye and color information to the other eye during inspection, even in the absence of binocular rivalry. Nor was it possible to induce depth-specific color aftereffects following an inspection period during which random-dot stereograms were viewed. with crossed and uncrossed disparity seen in different colored light. Masking and aftereffect in the perception of stereoscopic depth were also nonselective to color; in both cases, perceptual distortion was controlled by stereospatial variables but not by the color relationship between the inspection and test stimuli. The results suggest that binocularly driven spatial detectors in human vision are insensitive to wavelength.
\end{abstract}

There is now extensive psychophysical evidence suggesting that at least some detection mechanisms in human vision are responsive to both color and spatial attributes of the visual stimulus. In the initial study, McCollough (1965) found that orientation-specific color aftereffects could be induced by displaying alternately a horizontal grating in blue light and a vertical grating in orange light. When the gratings were subsequently viewed in white light, Ss reported that horizontal lines appeared orange and vertical lines looked blue. McCollough attributed these aftereffects to selective adaptation of neural detectors tuned to both orientation and wavelength. The assumption is that there are separate detectors for orange-vertical and blue-vertical lines, and defectors excited during the inspection period are suppressed for a period of time afterwards. The vertical test lines presented in white light are normally signaled by the relative response level of orange-vertical and blue-vertical detectors, but appear colored following inspection because one class of detector is in an adapted state. Color aftereffects that are specific to image motion (Hepler, 1968: Stromeyer \& Mansfield, 1970) and spatial periodicity (Breitmeyer \& Cooper, 1972; Lovegrove \& Over, 1972: Stromeyer. 1972) have been explained in similar terms.

Spatially linked color aftereffects (subsequently referred to as the McCollough effect) can be induced with either monocular or binocular inspection. However, the McCollough effect is not obtained when adaptation stimuli are viewed by one eye and test stimuli by the other eye (McCollough. 1965: Hepler. 1968: Murch, 1969; Stromeyer \& Mansfield, 1970). Such data suggest

\footnotetext{
*This study was supported by an award from the Australian Research Grants Committee. We thank Jack Broerse and Ann Marie Parker for their assistance in data collection.

$\div$ Requests for reprints should be sent to Ray Over. Department of Psychology, University of Queensland, St. Lucia. Australia 4067.
}

that neural detectors tuned to both color and spatial attributes have exclusively monocular inputs. This interpretation is supported by several recent studies that have examined color selectivity in spatial aftereffect and masking. Lovegrove, Over, and Broerse (1972) found that the motion aftereffect obtained when stationary test lines are seen following inspection of moving lines is maximal when the inspection and test lines are the same color and is reduced when they differ in color. The motion aftereffect generated with inspection by one eye transfers to the other eye, but color specificity in the aftereffect is now lost. These results indicate that some motion detectors are color selective and others are not. The interocular transfer data suggest that at least some achromatic motion detectors can be driven by either eye, whereas chromatic motion detectors receive exclusively monocular inputs. Similarly, color selectivity in periodicity (Murch, 1972) and orientation (Lovegrove \& Over, 1973) aftereffects generated with monocular inspection is lost when the adaptation and test stimuli are viewed by separate eyes, although there is interocular transfer of spatial information.

The four experiments that follow examined the claim that spatial and color properties of the visual stimulus are conjointly processed only by monocular detectors. Experiment I compared the McCollough effect with color and spatial information presented to the same eye and to separate eyes during inspection. In Experiment II, random-dot stereograms were inspected in colored light to establish whether disparity-specific color aftereffects occur, and Experiments III and IV investigated color selectivity in aftereffect and masking within stereoscopic depth perception.

\section{EXPERIMENT I}

This experiment considered whether the McCollough effect can be induced by simultaneously presenting 


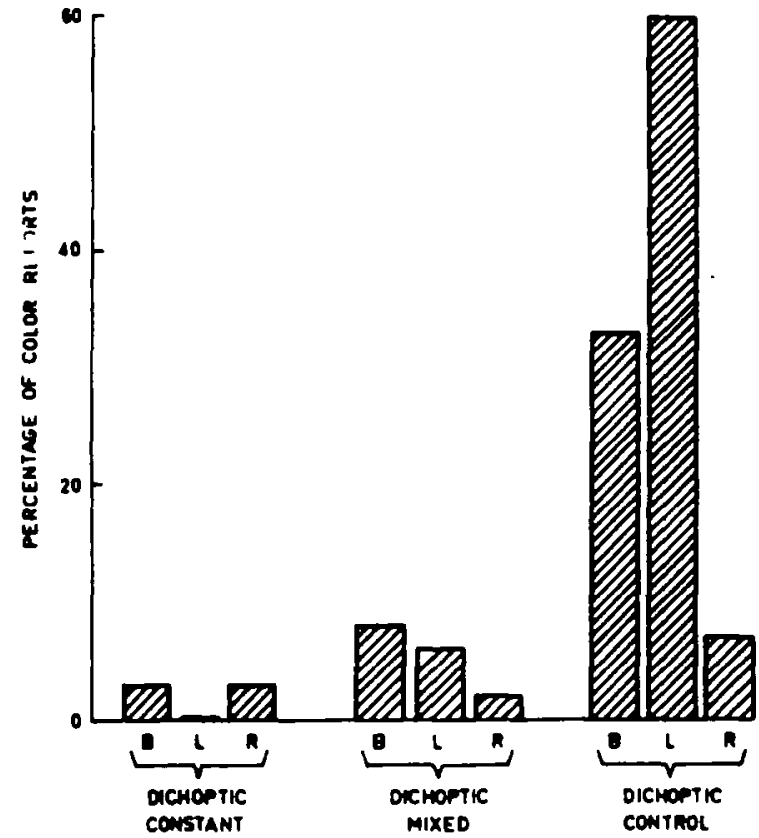

Fig. 1. Percentage of color reports as a function of adaptation conditions and with the test figure viewed binocularly (B), by the left eye alone (L), and by the right eye alone (R).

unpatterned color information to one eye and achromatic contour information to the other eye during inspection. The viewing conditions employed by Ono, Komoda, and Mueller (1971) to produce binocular color mixture were used to ensure that the dichoptically presented color and contour material were viewed without binocular rivalry. They found that different colors simultaneously presented to each eye for no longer than $100 \mathrm{msec}$ on each occasion resulted in binocular color mixture, but that with longer exposure there was binocular rivalry. Two conditions of dichoptic presentation were adopted. In one case, color information was always presented to one eye and contour information to the other eye (dichoptic constant condition), and in the other case, display of red light to the left eye and vertical lines to the right eye were alternated with presentation of horizontal lines to the left eye and green light to the right eye (dichoptic mixed condition). Following an adaptation period, Os were required to judge the color appearance of gratings presented in white light and viewed either monocularly or binocularly.

Orientation-specific color aftereffects should not occur following either form of dichoptic stimulation if the McCollough effect depends on adaptation of monocular detectors conjointly tuned to contour and color. However, the dichoptic mixed condition might result in eye-specific color afterimages unrelated to contour orientation. Perception was also studied with color and contour viewed by one eye while an unpatterned achromatic field was at the same time displayed to the other eye. The McCollough effect should occur under these conditions, but be reduced because the colored gratings seen during adaptation appeared desaturated. This control was employed because the dichootic conditions also required that desaturated color be seen during inspection.

\section{Method}

\section{Subjects}

The six Ss tested under the three induction conditions had normal vision and were selected because in each case the traditional MoCollough effect was readily induced under standard conditions.

\section{Materials and Procedure}

The adaptation and test stimuli were displayed by back-projection of filters and gratings in a Gerbrands three-field tachistoscope (Model T-3B-1). Polarizing filters were used to separate inputs to the two eves. During adaptation under a specific induction condition, $S$ viewed altemately either a red-vertical and a green-horizontal grating or a green-vertical and a red-horizontal grating. The spatial frequency of each grating was 4 cycles/deg, and the colors were produced using Wratten Filters 26 (dominant wavelength, $620.6 \mathrm{~nm}$ in Illuminant A) and 55 (dominant wavelength, $524.1 \mathrm{~nm}$ in. Illuminant A). The adaptation stimuli were alternated every 10 sec. Each stimulus was shown intermittently, with a dark interval of $100 \mathrm{msec}$ following each $100-\mathrm{msec}$ presentation: under these conditions, dichoptic display of color and contour resulted in a stable percept of a colored grating without binocular rivalry. The gratings subtended $4 \times 4 \mathrm{deg}$, and the space-average luminance of each monocular field was maintained at $18.0 \mathrm{~cd} / \mathrm{m}^{2}$.

Each S was tested under the three adaptation conditions (dichoptic constant, dichoptic mixed. dichoptic control) described earlier. The color-orientation combinations used during adaptation were counterbalanced between $S s$, and a Latin-square design determined the order in which Ss were tested across the three conditions. Measures were taken under one condition within a session, and a rest period of at least 2 days was left between sessions. The 20 -min adaptation period was interrupted by test probes taken after 5.10 .15 , and $20 \mathrm{~min}$. On a probe trial. $S$ viewed for $5 \mathrm{sec}$ juxtaposed vertical and horizontal gratings (subtending $4 \times 4$ deg overall) shown in white light. His task was to report whether either grating appeared colored and. if so, whether the color most resembled red or green. Measures were taken with the probe stimuli viewed binocularly: with the left eye alone, and with the right eye alone. In each case, the vertical grating was located on the left of the display on one trial and on the right side on a further trial. The order of testing across probe conditions was randomized between Ss.

\section{Results and Discussion}

Figure 1 shows the percentage frequency of color reports on probe trials under the three induction conditions. Values are shown separately for binocular. left-eye-alone, and right-eye-alone presentations of the test gratings, but results have been pooled across the other conditions (period of adaptation. color-contour combinations. relative position of the target grating). Color reports were selective to contour orientation only under the dichoptic control condition when color and 
contour had been simultaneously presented to the left ey'e during inspection. The McCollough effect was consistently induced in the left eye, but not in the right eve, which had been stimulated by an unpatterned achromatic field. On binocular test judgments, the $\mathrm{McC}$ ollough effect occurred with lower frequency and appeared desaturated. Neither the dichoptic constant nor the dichoptic mixed conditions produced the McCollough effect, and the color reports that occurred with low frequency on test trials were not systematically related to the properties of the inspection stimuli.

Under all three adaptation conditions, the Ss viewed what appeared to be colored gratings, but in two cases the phenomenal appearance was produced by synchronously pulsing a homogeneous colored field to one eye and an achromatic grating to the other eye. The present data indicate that the phenomenal properties of the adaptation stimuli are not responsible for generation of the McCollough effect; instead, color and contour information must be displayed to the same eye during the adaptation period. It follows that dichoptic display of color and contour had different neural consequences than monoptic presentation. In terms of the logic so far developed, the results of the present experiment suggest that in human vision detectors conjointly tuned to wavelength and spatial orientation receive exclusively monocular inputs.

\section{EXPERIMENT II}

The random-dot technique that has been widely used by Julesz (1971) permits determination of whether color aftereffects specific to stereoscopic depth can be induced. By this method, the two eyes simultaneously view separate random-dot patterns, which are identical except that a group of elements has been laterally shifted in spatial position to one eye relative to the other eye. On binocular fusion, the translated elements appear clustered as a cohesive figure in front of or behind (depending on the direction of image difference between the two eyes) the unshifted surround. Space perception in this situation must reflect the response of neural mechanisms tuned to binocular disparity in that neither figure nor depth is visible if either random-dot pattern is viewed monocularly. Several accounts (e.g., Bishop \& Henry, 1971; Julesz, 1971) have been offered of neural mechanisms mediating stereospatial perception.

The claim under examination is that detectors mediating the McCollough effect receive exclusively monocular inputs. The expectation, therefore, is that exposure to a binocularly" induced "near" figure in red light and "far" figure in green light does not result in disparity-selective color aftereffects when the displays are later viewed in white light.

\section{Method}

Photographic negatives of a random-dot stereopair (taken from Julesz. 1971, p. 268) were back-illuminated. and polarizing filters used to separate the inputs to the two eyes. Binocular fusion was assisted by prisms (4.25 diopters) located in the eyepiece. On fusion of the stereopair. S saw two rectangles, one located above and the other below a central fixation marker. In terms of visual depth. one rectangle appeared in front of and the other behind the marker. The total display subtended 22 deg $12 \mathrm{~min} \times 22 \mathrm{deg} 12 \mathrm{~min}$. and each rectangle subtended $5 \mathrm{deg}$ $36 \mathrm{~min}$ (vertical) $\times 6 \mathrm{deg} 14 \mathrm{~min}$ (horizontal). The fixation marker was a bar. $16 \times 30 \mathrm{~min}$. positioned to provide 13 minarc of crossed disparity relative to the background elements. The elements comprising one rectangle (near) differed between the stereopair by 16.9 minarc of crossed disparity and in the case of the other rectangle (far) by 9.1 minarc cross disparity. The $E$ could readily adjust the stimuli so that one or both rectangles were visible at the one time, with either rectangle shown above or below the central marker.

There were two stages of testing. In the tirst stage, four Ss with experience in stereoscopic perception of random-dot patterns and in induction of the standard McCollough effect viewed in succession one stereoscopically produced rectangle in red light and the other rectangle in green light. Presentations lasted $10 \mathrm{sec}$ and were separated by a dark interval of $2 \mathrm{sec}$. The test stimuli (the two stereopairs in white light) were shown after 10 and $20-\mathrm{min}$ adaptation, and $S$ was required to judge the color appearance of each rectangle. For half of each 2-min test probe, the relative location (above or below the central marker) of each rectangle was reversed relative to the adaptation positions. This method ensured that the $\mathrm{I}$ (cCollough effect would not be confounded with complementary afterimages of the color presented at each location.

In the second stage, the two rectangles were simultaneously presented during adaptation, with the near rectangle shown in green light and the far rectangle in red light. The display was internupted every $10 \mathrm{sec}$ by a dark interval of $2 \mathrm{sec}$. Adaptation continued for $40 \mathrm{~min}$. and the two $\mathrm{Ss}$ then judged the depth and color appearance of the rectangles in white light. Judgments were made with the rectangles in the same and in the reversed spatial positions relative to adaptation.

\section{Results and Discussion}

An analog of the McCollough effect could not be produced within stereoscopic depth perception, in that no $S$ in either the first or second stage reported that the upper and lower test rectangles differed in color appearance. However, both Ss in the second stage reported that the test rectangles merged into the background when there was careful fixation of the central marker. In such cases, the Ss reported complementary retinopic colored afterimages, which in turn faded when stereopsis returned.

Generation of the McCollough effect requires that the adaptation stimuli be signaled within the visual system by separate neural channels (see Fidell, 1970: Lovegrove \& Over, 1972). It is not possible to establish directly whether the near and far rectangles displayed in different colored light during adaptation were represented by separate populations of detectors. However, in the present experiment, the stereostimuli paired with color differed in disparity by 7.8 minarc, and Long and Over have demonstrated, by use of masking paradigms, that channels mediating fine stereopsis are tuned over less than 4 minarc. It is also unlikely that the temporal values (adaptation period. alternation rate. interstimulus dark interval) adopted in 
the experiment operated against generation of disparity-specific color aftereffects in that the conditions were well within the range in which color aftereffects selective to orientation, motion, and periodicity can be produced.

The further possibility is that the human visual system does not possess detectors that respond to wavelength as well as stereospatial information. This interpretation is consistent with the evidence that color selectivity is not maintained in the tilt (Lovegrove \& Over, 1973). motion (Lovegrove, Over, \& Broerse, 1972), and periodicity (Murch, 1972) aftereffects when the inspection figure is viewed by one eye and the test figure by the other ey'e. Interocular transfer of aftereffect can occur only to the extent that neural detectors adapted as a function of inspection are normally involved in representation of the test stimulus. The loss in color selectivity with interocular transfer suggests that color-tuned spatial detectors are limited to binocular processing, while monocular units are tuned to color but not to spatial at tributes. This proposal gains support from two recent studies. Ramachandran and Sriram (1972) found that depth perception with random-dot stereograms is unimpaired when the two eyes view the pattern in different colored light. Lu and Fender (1972) have shown that stereoscopic depth perception is lost when the elements in a random-dot stereogram differ in wavelength but are identical in luminance: this loss in depth perception occurs even though spatial disparity within the stereogram has been unaffected. Both data suggest that wavelength and binocular disparity are processed at different sites in the visual system.

\section{EXPERIMENT III}

Successive presentation of random-dot stereograms containing different disparity information produces an aftereffect in binocular depth perception (Blakemore \& Julesz, 1971: Long \& Over, 1973). The aftereffect takes the form of spatial repulsion, with the binocular target appearing nearer to $S$ following exposure to uncrossed disparity and farther away following exposure to crossed disparity.

Spatial aftereffects have been attributed to selective adaptation of finely tuned neural detectors (see Coltheart. 1971: Over, 1971). The assumption is that the perceived spatial position of the target is determined by the mean of the distribution of input in terms of which the stimulus is represented in the visual system. It is further proposed that detectors excited by the inspection stimulus remain in an adapted state immediately afterwards. Thus, the distribution by which the target is represented is symmetrically inhibited when the inspection and target stimuli are identical in spatial value: as the mean is not shifted. the position of the target is not misperceived. There is asymmetrical inhibition when the inspection and target stimuli differ: the mean of the distribution is shifted. and the target appears displaced in the opposite direction to the inspection figure.

It was earlier proposed that color selectivity in spatial aftereffects implies that color and spatial information are processed by the same detection mechanism. In the present experiment. the stereoscopic depth aftereffect was measured by determining the disparity needed within the test stereopair to cancel the shift in perceived depth induced by prior exposure to different levels of stereospatial information. The hypothesis that binocularly driven spatial detectors in human vision are insensitive to color was tested by establishing whether the stereoscopic aftereffect is dependent on the color relationship between the inspection and test stimuli.

\section{Method}

\section{Subjects}

Four male Ss with normal color vision and spatial acuity received $2 \mathrm{~h}$ of preliminary practice in judging relative depth when achromatic random-dot stereograms containing varied amounts of disparity were briefly shown.

\section{Waterials and Procedure}

Random-dot stereograms were used to vary the visual depth of a center square within the inspection and test stimuli. In the basic pair of stereostimuli (zero disparity). the left and right fields consisted of $960 \times 960$ randomly positioned black and white dots, and on fusion the binocular field subtended 5 deg verticaly and horizontally. All other stereopairs differed in terms of the relative lateral displacement of a 2-deg center square in the two fields. When displaced nasally. it appeared centrally in front of the plane of the background, and with temporal displacement, it was perceived as farther away than the background. Stereopairs covering the range from 8 minarc of crossed disparity to 8 minarc of uncrossed disparity in 40-secarc steps were used. Photographic transparencies of each stereopair were rear-projected to the appropriate eye by Polaroid filters. Two prisms (4.25 diopters) were inserted into the eyepiece to aid binocular fusion. and there was also a constantly presented fixation point viewed by both eyes and centrally located in the same visual plane as the background to the square.

Wratten filters (Nos. 26 and 55) were used to vary the color of the inspection and test stimuli. and space-arerage luminance was maintained at $2.50 \mathrm{~cd} / \mathrm{m}^{2}$. The magnitude of the aftereffect was measured by a cancellation method (Long \& Over. 1973). On each trial, $S$ initiated the display when fixation was at tained. and the sequence was inspection stimulus $(250 \mathrm{msec})$. dark interval $(10 \mathrm{msec})$. and test stimulus $(250 \mathrm{msec})$. The S's task was to report whether the central square of the test stimulus appeared in front of or behind its background. Lnder a given inspection condition. the test stimulus initially contained zero disparity. and over successive trials. disparity was varied in 40-secarc step; in accord with double-random staircase procedures until six reversals in judgment occurred. Measures were obtained for red-red. red-green. green-green. and green-red combinations of inspection and test stimuli, and for two depth values 10 and 6 minarc of crossed disparity) of the inspection stimulus. The sequence of testing across color conditions was counterbalanced between Ss. and there was a rest period of $5 \mathrm{~min}$ following testing under each condition.

\section{Results and Discussion}

Figure 2 shows the proportion of trials on which the 
test stimulus at its different disparity values was judged as behind the surround after exposure to the 0 - and 6-minarc inspection stimuli. Values are given separately for the four color combinations of inspection and test stimuli. The aftereffect induced by exposure to 6-minarc disparity is given by the shift in perceived depth relative to measures under the 0 disparity (control) condition. For purposes of statistical analysis, the location at which the test square appeared in the same visual plane as its background was calculated under each inspection condition as the disparity midway between values on which $S$ reversed depth judgments. An analysis of variance based on these measures indicated that the perceived location of the test stimulus varied significantly as a function of the extent of binocular disparity within the inspection stereogram, $F(1,3)=$ $11.56, p<.05$. The color relationship between the inspection and test stereograms, $F(3,9)=0.44, p>.05$, and the interaction between inspection disparity and color combination, $F(3,9)=0.94, p>.05$, did not exert significant influence.

The results show that the magnitude of the stereoscopic depth aftereffect remains unchanged by a shift in color between inspection and adaptation. The stereospatial aftereffect differs from tilt, periodicity, and motion aftereffects in this regard, and the present data reinforce the claim made earlier that binocular depth and color are processed in human vision by separate detection mechanisms.

\section{EXPERIMENT IV}

The visibility of a binocular target is impaired if $S$ has previously viewed an inspection stimulus containing similar stereospatial information (Long \& Over ${ }^{1}$ ). In neural terms, a target can be detected only when the input it generates is critically above the noise level of the transmission system. If detectors normally engaged in representation of the stimulus are in an adapted state as a result of inspection, the signal-to-noise ratio critical for detection can be maintained only by an increase in the energy level of the target. In these terms, one stimulus can mask another only to the extent that the two stimuli are normally processed by the same neural detectors. The argument so far developed is that compound color and spatial processing in human vision is restricted to monocular input. If masking in the perception of stereoscopic depth results from selective adaptation of neural detectors tuned to binocular disparity, the masking function should be unaffected by the color relationship between inspection and test stimuli. This hypothesis is tested in Experiment IV.

\section{Method}

\section{Subjects}

The four Ss who had served in Experiment III were tested.

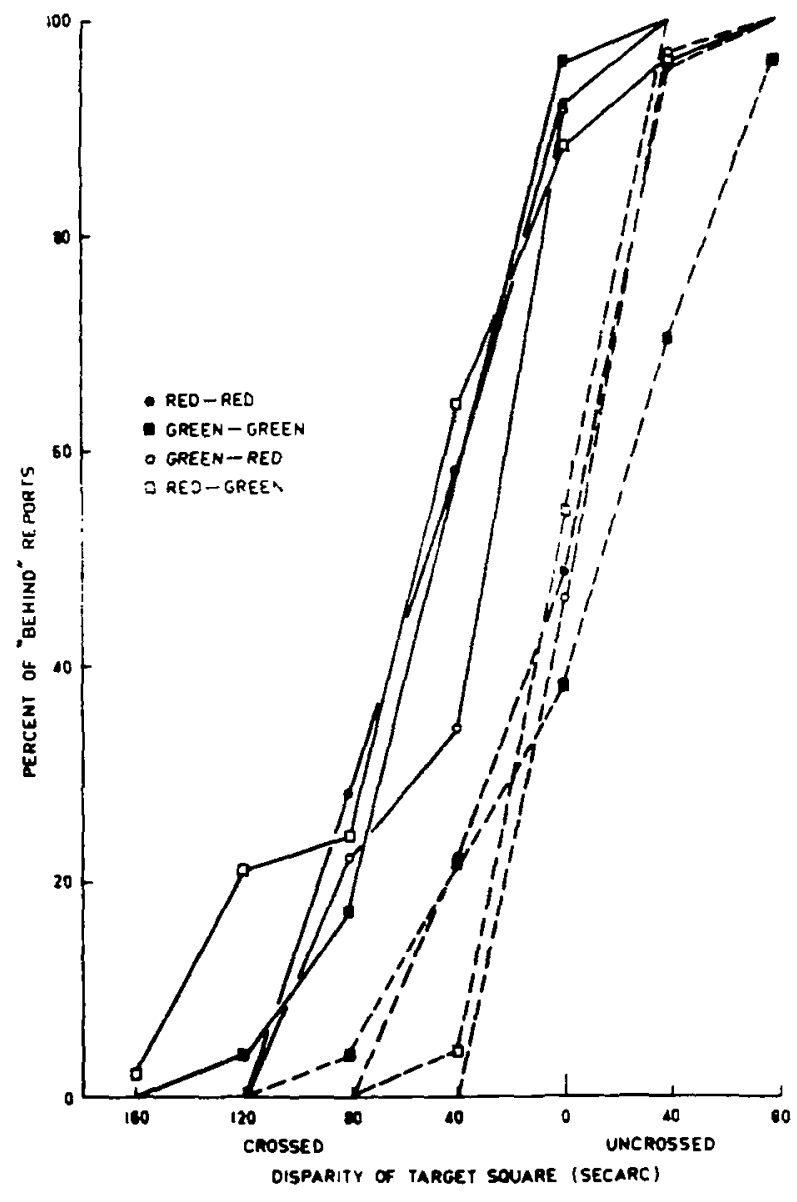

Fig. 2. Depth judgments as a function of the color and disparity relationships between the inspection and test stimuli. The inspection disparities were 0 minarc $\left(--_{-}\right)$and 6 minarc (-).

\section{Naterials and Procedure}

Forward masking was studied, and the stimulus sequence on each trial was: fixation point $(1 \mathrm{sec})$. inspection stimulus $(250 \mathrm{msec})$. dark interval $(10 \mathrm{msec})$. test stimulus $(105 \mathrm{msec})$. The test stereogram presented either 0 disparity (catch presentation) or a center square formed by 6 minarc of crossed disparity (target presentation). The $S$ 's task on each trial was to report which test condition has been displaced. Detection was measured by a blockwise tracking method (Over. Broerse. \& Crassini, 1972). Within a block of 12 trials, six target and six catch presentations were given in random order at a specific luminance level. If $S$ was more (less) than 75 ? correct in his reports over the total block. the luminance of the test stimulus was decreased (increased) in logarithmic steps (.1. .2. .4, $.8 \mathrm{~cd} / \mathrm{m}^{2}$ ) until $75^{\circ}$ accuracy was attained in a block or bracketed by successive blocks.

Detection was studied as a function of the depth information provided in the inspection stereogram (2. 4.6. and 8 minarc of crossed disparity) and the color combination of inspection and test stimuli (red-red. red-green. green-green. green-red). Color was controlled using Wratten filters (Xos. 26 and 55), and the space-average luminance of the inspection stereogram was maintained at $2.50 \mathrm{~cd} / \mathrm{m}^{2}$. Latin squares were used in rarying 


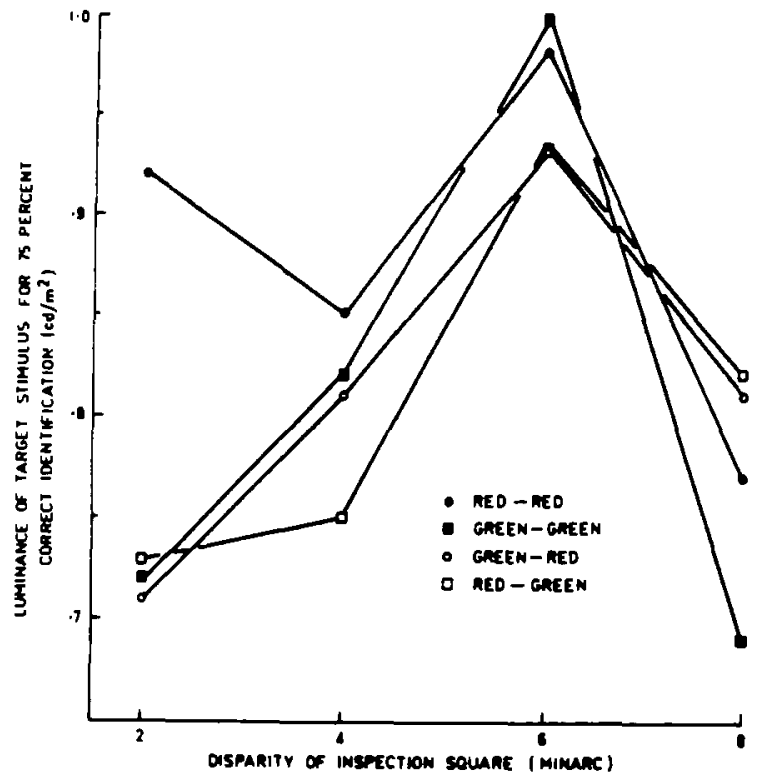

Fig. 3. Visibility of target square for different color combinations of inspection and target stereograms.

the sequence of testing disparity values and color combinations across Ss.

\section{Results and Discussion}

The mean space-average luminance required for $75 \%$ accuracy in identification of the test stimulus following exposure to each inspection stereogram is shown in Fig. 3. The threshold for detection varied significantly as a function of the disparity level during inspection, $F(3,9)=9.21, p<.01$, but did not differ across color combinations, $F(3,9)=0.26, p>.05$. The interaction between disparity and color was not significant, $F(9,27)$ $=1.86, \mathrm{p}>.05$. The probability of accepting the null hypothesis when it is false is less than $1 \%$ using the method described by Kirk (1972, p. 109). Comparisons between the two-way interaction means by Duncan's multiple range test showed that masking was disparity-selective and extended over a range of only 4 minarc. This is the same value as found by Long and Over with achromatic stereograms. The peak masking values obtained for the different color combinations did not differ significantly: this result is consistent with the earlier claim that disparity channels in human vision are insensitive to color.

\section{GENERAL DISCUSSION}

Several psychophysical paradigms have demonstrated that color-spatial linkages are restricted to monocular visual processing. The McCollough effect can be readily generated when both contour and color are seen by either one eye or both eyes during inspection, but color aftereffects resulting from monoptic induction do not show interocular transfer. Under dichoptic conditions of inspection, the McCollough effect is not obtained when contour information is presented to one eye and color information to the other eye during inspection, even in the absence of binocular rivalry. Nor is it possible to produce depth-specific color aftereffects by adaptation to crossed disparity in red light and uncrossed disparity in green light. In addition, masking and aftereffect in the perception of stereoscopic depth are also nonselective to color: in both cases, perceptual distortion is under the control of stereospatial variables and is unaffected by a shift in color between inspection and test.

In terms of the general argument that the McCollough effect occurs through selective adaptation of feature detectors, the present data suggest that binocularly driven spatial analyzers in human vision are insensitive to color, whereas at least some monocularly driven analyzers are tuned to both color and spatial attributes. Claims about information processing developed from psychophysical data are inevitably indirect, but the alternative neural logics that might underlie specific perceptual effects can be limited by reference to physiological evidence. Microelectrode studies employing achromatic gratings have shown that single cells in the cat and monkey visual cortex are selective to contour orientation, motion velocity and direction, and binocular image disparity (see Blakemore, Iversen, \& Zangwill, 1972). Lennox-Buchthal (1962) isolated cells with narrow-band spectral sensitivities in the monkey cortex. but the use of unpatterned colored fields as probe stimuli did not allow spatial tuning to be investigated.

Hubel and Wiesel (1968) studied cortical response to colored oriented slits, and located color-spatial. color-alone, and spatial-alone cells. All measures were taken with monocular stimulation. Cells selective to both color and orientation were isolated with low frequency, but this may have reflected the fact that the majority of cells under study had receptive fields outside the fovea. Gouras (1972) has used monochromatic slits to establish the response of monocularly stimulated cells in the foveal striate cortex of the rhesus monkey. Of 162 cells, $46 \%$ were tuned to orientation but not to color, $28 \%$ to both orientation and color, and $26 \%$ to color but not to orientation.

There is an obvious noed for classification of cells during binocular as well as monocular stimulation. The results of the present experiments provide guidelines for physiologists by suggesting that color-spatial linkages are limited to monocular processing.

\section{REFERENCES}

Bishop, P. O., \& Henry, G. H. Spatial vision. Annual Review of Psychology, 1971, 22. 119-160.

Blakemore. C.. \& Julesz. B. Stereoscopic depth aftereffect produced without monocular cues. Science. 1971. 71. 286-288. 
Blakemore, C., Iversen, S. D., \& Zangwill, O. L. Brain functions. Annual Review of Psychology, 1972, 23, 413-456.

Breitmeyer, B. G., \& Cooper, L. A. Frequency-specific color adaptation in the human visual system. Perception \& Psychophysics, 1972, 11, 95-96.

Coltheart, $M$. Visual feature-analyzers and aftereffects of tilt and curvature. Psychological Review, 1971, 78, 114-121.

Fidell, L. S. Orientation specificity in chromatic adaptation of human "edge" detectors. Perception \& Psychophysics, 1970, 8, 235-237.

Gouras, P. Color opponency from fovea to striate cortex. Investigative Ophthalmology, 1972, 11, 427-434.

Hepler, N. Color: A motion-contingent aftereffect. Science, $1968,162,376-377$.

Hubel, D. H., \& Wiesel, T. N. Receptive fields and functional architecture of monkey striate cortex. Journal of Physiology, 1968, 195, 215-243.

Julesz, B. Foundations of cyclopean perception. Chicago: University of Chicago Press, 1971.

Kirk, R. Experimental design: Procedures for the behavioral scientist. Belmont, Calif: Wadsworth, 1972.

Lennox-Buchthal, M. A. Single units in monkey (Cercocebus torquatus atys) cortex with narrow spectral responsiveness. Vision Research, 1962, 2, 1-15.

Long, N., \& Over, R. Stereoscopic depth aftereffect with random-dot patterns. Vision Research, 1973, in press.

Lovegrove, W. J., \& Over, R. Color adaptation of spatial frequency detectors in the human visual system. Science, $1972,176,541-543$.

Lovegrove, W. J., \& Over, R. Color selectivity in orientation masking and aftereffect. Vision Research, 1973, in press.

Lovegrove, W. J., Over, R., \& Broerse, J. Color specificity in the motion aftereffect. Nature, 1972, 238, 334-335.

Lu, C., \& Fender, D. The interaction of color and luminance in stereoscopic vision. Investigative Ophthalmology, 1972, 11, 482-489.

McCollough, C. Color adaptation of edge-detectors in the human visual system. Science, 1965, 149, 1115-1116.

Murch, G. M. Size judgments of McCollough afterimages. Journal of Experimental Psychology, 1969, 81, 44-48.

Murch, G. M. Binocular relationships in a size and color orientation-specific aftereffect. Journal of Experimental Psychology, 1972, 93, 30-34.

Ono, H., Komoda, M., \& Mueller, E. R. Intermittent stimulation of binocular disparate colors and central color fusion. Perception \& Psychophysics, 1971, 9, 343-347.

Over, R. Comparison of normalization theory and neural enhancement explanation of negative aftereffects. Psychological Bulletin, 1971, 75, 225-243.

Over, R., Broerse, J., \& Crassini, B. Orientation illusion and masking in central and peripheral vision. Journal of Experimental Psychology, 1972, 96, 25-31.

Ramachandran, V. S., \& Sriram, S. Stereopsis generated with Julesz patterns in spite of rivalry imposed by coloured filters. Nature, 1972, 237, 347-348.

Stromeyer, C. F. Edge-contingent color after-effects: Spatial frequency selectivity. Vision Research, 1972, 12, 717-733.

Stromeyer, C. F., \& Mansfield, R. J. W. Colored aftereffects produced with moving edges. Perception \& Psychophysics, 1970, 7, 108-114.

\section{NOTE}

1. Long, N., \& Over, R. Disparity determinants of stereoscopic depth masking. Submitted for publication.

(Received for publication November 22, 1972; revision received February 1, 1973.) 